\title{
Study on Nonlinear Dynamics and Chaos Suppression of Active Magnetic Bearing Systems Based on Synchronization
}

\author{
Shun-Chang Chang \\ Department of Mechanical and Automation Engineering, Da-Yeh University, No. 168 University Road, Dacun, \\ Changhua 515006, Taiwan \\ Correspondence should be addressed to Shun-Chang Chang; changsc@mail.dyu.edu.tw
}

Received 21 January 2021; Revised 20 March 2021; Accepted 8 April 2021; Published 21 April 2021

Academic Editor: Renming Yang

Copyright (C) 2021 Shun-Chang Chang. This is an open access article distributed under the Creative Commons Attribution License, which permits unrestricted use, distribution, and reproduction in any medium, provided the original work is properly cited.

\begin{abstract}
This study employed a variety of nonlinear dynamic analysis techniques to explore the complex phenomena associated with a nonlinear mathematical model of an active magnetic bearing (AMB) system. The aim was to develop a method with which to assume control over chaotic behavior. The bifurcation diagram comprehensively explicates rich nonlinear dynamics over a range of parameter values. In this study, we examined the complex nonlinear behaviors of AMB systems using phase portraits, Poincaré maps, and frequency spectra. Furthermore, estimates of the largest Lyapunov exponent based on the properties of synchronization confirmed the occurrence of chatter vibration indicative of chaotic motion. Thus, the proposed continuous feedback control approach based on synchronization characteristics eliminates chaotic oscillations. Finally, some simulation results demonstrated the feasibility and efficiency of the proposed control scheme.
\end{abstract}

\section{Introduction}

Active magnetic bearings (AMBs) are widely used in rotating machinery and have high potential for machining applications. These noncontact bearings use magnetic forces to firmly hold the rotor and maintain separation between the rotor and the stationary components. In such a linear case study, the electromagnetic force is linearized about the operating point. In AMB systems, the occurrence of large unbalanced forces in rotor bearings can cause nonlinear motions of high amplitude. However, a closed-loop control system is required to stabilize the system by eliminating vibrations caused by disturbing forces. AMBs are regarded as inherently nonlinear due to nonlinearities of the electromagnetic forces. Accurate control of the system requires that designers account for the effects of nonlinearities. Thus, these complex nonlinear dynamics must be considered for in the design of AMB systems. Accordingly, in the present author's previous work [1], an experiment involving an unloaded symmetric rotor with a flexible coupler at one end and a bearing comprising two pairs of electromagnets at the other end (Figure 1) was conducted by applying a series of nonlinear electromagnetic forces to identify a nonlinear model of this system. Figure 2 illustrates the block diagram of the AMB control system along the horizontal direction. In the aforementioned study, we sought to predict these nonlinear dynamics by modifying the conventional identification technique based on the principle of harmonic balance to characterize the system with a higher degree of precision.

This model successfully captured the primary characteristics of the system by comparing the simulated frequency responses with those obtained experimentally [1]. However, theoretical analyses of this model to determine whether the nonlinear mathematical model derived experimentally can predict and characterize the dynamics of the physical system have not yet been undertaken. Various works have been carried out to study the chaotic motion in AMB systems [2-6]. But the chaotic motion that occurs when the rotor strikes the electromagnet that using the identification of a mathematical model for the AMB system has not been examined. 


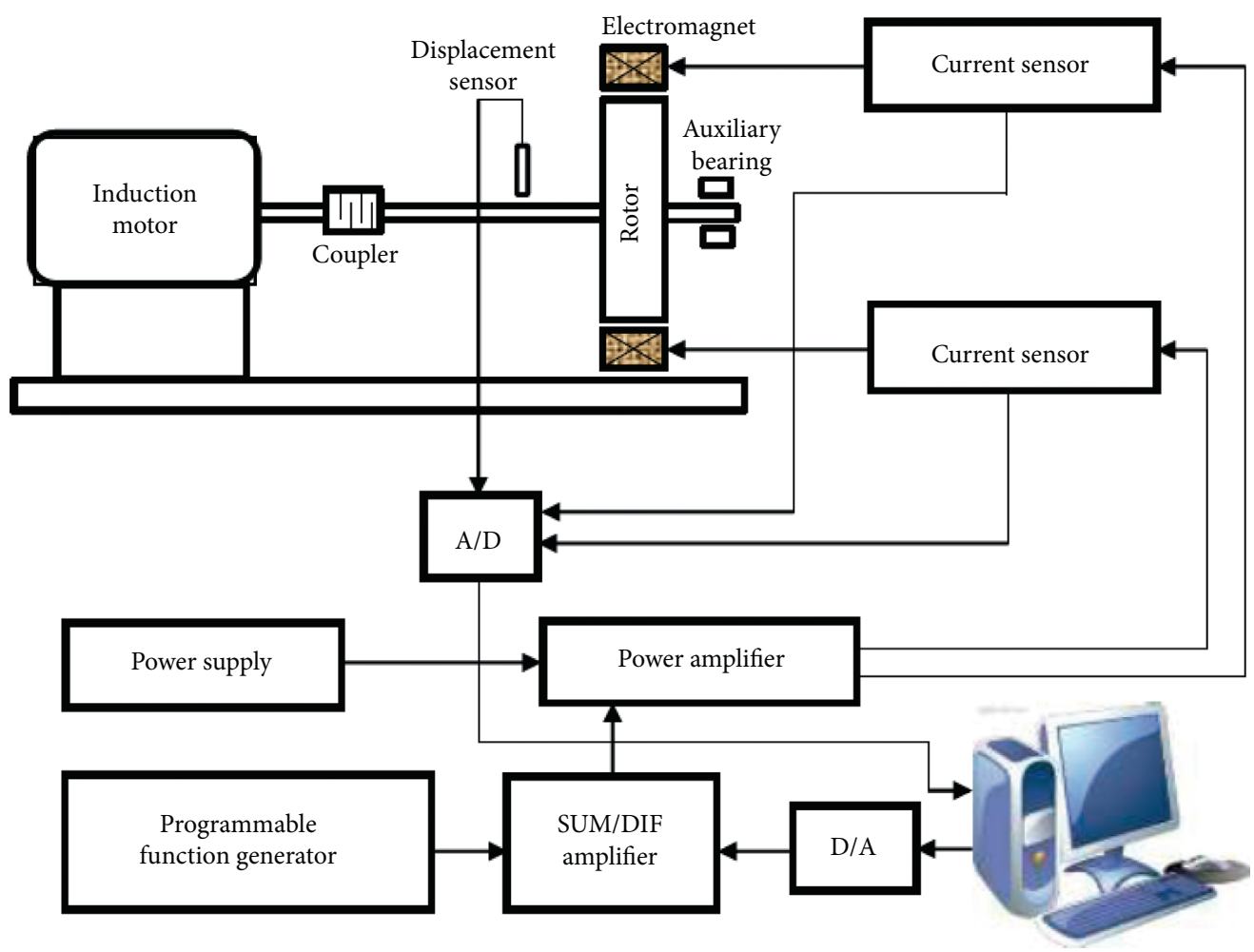

FIgURE 1: Schematic diagram of active magnetic bearing systems.

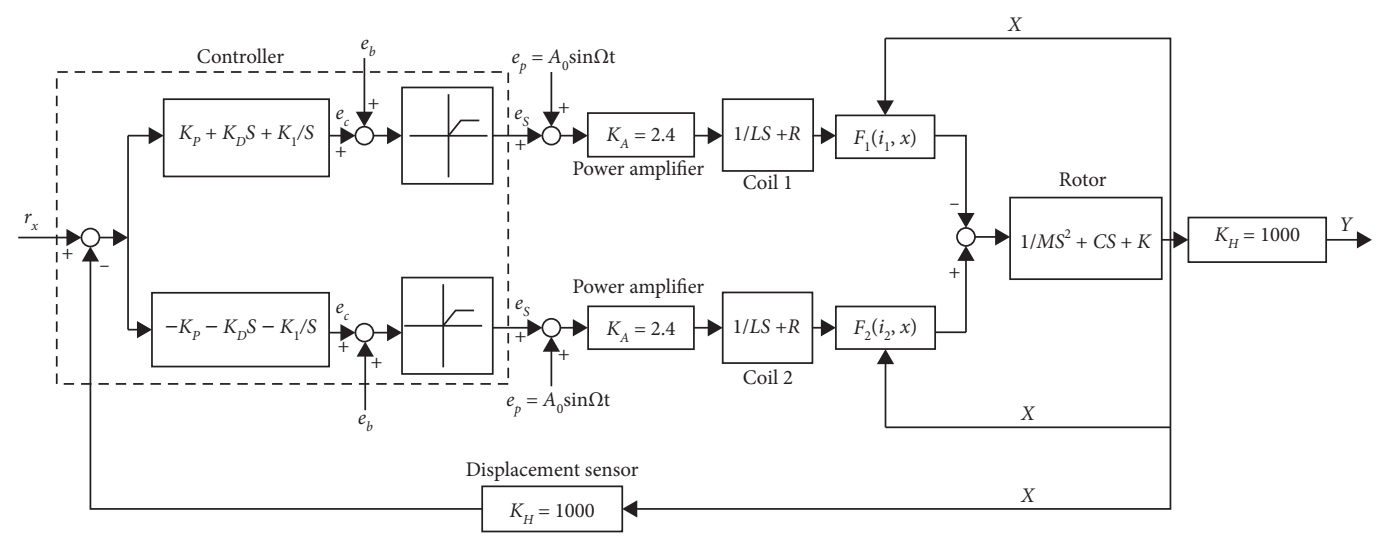

Figure 2: An overall block diagram of the AMB control system along the horizontal direction.

In this study, we used bifurcation diagrams, phase portraits, Poincaré maps, frequency spectra, and Lyapunov exponents to observe periodic orbits, bifurcation, and chaotic phenomena in an identified mathematical model of the AMB system. Across a broad range of parameters, the Lyapunov exponent offers the most powerful methods to measure the sensitivity of a dynamic system with regard to its initial conditions. This approach can be used to determine whether a system is susceptible to chaotic motion. The algorithms for computing Lyapunov exponents of smooth dynamic systems are well established [7-10]. However, a number of nonsmooth dynamic systems possess discontinuities, for example, discontinuities associated with dry friction, backlash, and saturation for which these algorithms cannot be applied directly. Numerous studies have developed procedures for the calculation of Lyapunov exponents associated with nonsmooth dynamic systems [11-13]. In this study, we adopted the method developed by Stefanski [13] for estimating the largest Lyapunov exponent for AMB systems with closed-loop control.

Several practical engineering problems involving chaos require control techniques to convert chaotic attractors into stable periodic orbits. Since the pioneering work of Ott et al. [14] on controlling chaos, various modified methods and other approaches have been proposed [15-22]. Improving the performance of a magnetic bearing system necessitates the transformation of chaotic motion into a steady-state periodic orbit by the state feedback control and the injection of dither signals [22]. Accordingly, a continuous feedback control method, based on the synchronization properties 
proposed by Kapitaniak [18] and Pyragas [19], was used. Such schemes convert chaotic motion into stable motion by using feedback combined with a periodic external force. Simulation results demonstrate the efficacy of the proposed approach mechanism.

This paper is organized as follows. Section 2 describes an AMB system model and describes the complex dynamic behavior of a power system, which was elucidated using numerical analysis methods, such as bifurcation diagrams, phase portraits, Poincaré maps, and frequency spectra. Section 3 presents the Lyapunov exponent used to determine whether the system exhibits chaotic motion. A synchronization control technique for controlling chaos in the AMB system is described in Section 4. Conclusions are outlined in Section 5 .

\section{Overall Characteristics of AMB System: Simulations and Discussion}

The resulting nonlinear model of the AMB system [1] is as follows:

$$
\begin{aligned}
\dot{x}_{1}= & x_{2}, \\
\dot{x}_{2}= & -d_{1} x_{2}-d_{2} x_{1}+d_{3} x_{3}+d_{4} x_{4}+d_{5} x_{1}^{2} \\
& +d_{6} x_{1} x_{3}+d_{7} x_{1} x_{4}+d_{8} x_{3}^{2}+d_{9} x_{4}^{2}+d_{10} x_{1}^{3}, \\
L \dot{x}_{3} & +R x_{3}=K_{A}\left(e_{c 1}+A_{0} \sin \Omega t\right), \\
L \dot{x}_{4} & +R x_{4}=K_{A}\left(e_{c 2}+A_{0} \sin \Omega t\right),
\end{aligned}
$$

where

$$
\begin{aligned}
& e_{c 1}=K_{H}\left(K_{P} x_{1}+K_{D} x_{2}+K_{I} \int x_{1} \mathrm{~d} t\right), \\
& e_{c 2}=K_{H}\left(-K_{P} x_{1}-K_{D} x_{2}-K_{I} \int x_{1} \mathrm{~d} t\right),
\end{aligned}
$$

with the limitations

$$
\begin{gathered}
-3.5 \mathrm{~V}<e_{c 1}<6.5 \mathrm{~V}, \\
-3.5 \mathrm{~V}<e_{c 2}<6.5 \mathrm{~V}, \\
0.0 \mathrm{~A}<x_{3}<2.0 \mathrm{~A}, \\
0.0 \mathrm{~A}<x_{4}<2.0 \mathrm{~A},
\end{gathered}
$$

where $x_{1}$ is the displacement of the rotor around the equilibrium point, $x_{2}$ is the velocity of the rotor, $x_{3}$ and $x_{4}$ are coil currents oscillating around the bias current, $e_{c 1}$ and $e_{c 2}$ are the outputs of the proportional-integral-derivative controller to the two coils, $K_{P}=-55, K_{D}=-0.3$, and $K_{I}=-50$ are the control gains, $K_{A}(=2.4)$ and $K_{H}(=10000)$ represent the gains of the power amplifier and displacement sensor, and $A_{0} \sin \Omega t$ is the forcing voltage generated by a programmable function generator. The procedures used to derive the other coefficients necessary for (1a)-(1d) are listed in Table 1.

Numerical simulations based on (1a)-(1d) were performed to clarify the characteristics of the proposed system.
TABLE 1: Identified results of parameters for the AMB system.

\begin{tabular}{lc}
\hline System parameter & Identified value \\
\hline$d_{1}$ & 3.066133 \\
$d_{2}$ & $4.3315 \times 10^{3}$ \\
$d_{3}$ & -5.5786683 \\
$d_{4}$ & 6.5562 \\
$d_{5}$ & $1.43371 \times 10^{7}$ \\
$d_{6}$ & $-2.531 \times 10^{4}$ \\
$d_{7}$ & $-4.53203 \times 10^{4}$ \\
$d_{8}$ & 0.34514 \\
$d_{9}$ & -0.40171 \\
$d_{10}$ & $-2.399816 \times 10^{10}$ \\
$L$ & 0.0161203 \\
$R$ & 14.3128231 \\
\hline
\end{tabular}

In (1a)-(1d), the amplitude of the input excitation, $A_{0}$, equals $3.5 \mathrm{~V}$. The commercial software package DIVPRK of IMSL [23] in Fortran subroutines for mathematics was used to solve these ordinary differential equations. The resulting bifurcation diagram is presented in Figure 3; this figure clearly demonstrates that the first period-doubling bifurcation occurred at approximately $\Omega=19.5 \mathrm{~Hz}$ and chaotic motion appears at approximately $\Omega=18.63 \mathrm{~Hz}$. The perioddoubling route to chaos has been observed in Figure 3. Additional details of the responses exhibited by the system are presented in Figures 4-7, in which each type of response is characterized by time response, phase portrait, Poincaré map, and frequency spectrum. Figures $4(\mathrm{a})-4$ (d) prove that the $T_{f}$-period includes a constant term and the fundamental components. From Figures 5 and 6, we inferred that a cascade of period-doubling bifurcations produced a series of subharmonic components, revealing bifurcations with new frequency components at $1(\Omega / 2),(3 \Omega / 2),(5 \Omega / 2)$, and so on. The essence of chaotic behavior can be described using Poincaré maps, which present an infinite set of points, referred to as a strange attractor. Chaotic motion also exhibits a broad continuous frequency spectrum. Thus, strange attractors and continuous-type Fourier spectra are generally regarded as strong indicators of chaos, as illustrated in Figures $7(a)-7(d)$.

\section{Analysis of Chaotic Phenomena in AMB}

The largest Lyapunov exponent is a useful diagnostic index of the chaotic system. Every dynamic system possesses a spectrum of Lyapunov exponents $(\lambda)$, which determine length, area, and volume changes in phase space. In simple terms, Lyapunov exponents measure the rate of divergence (or convergence) between two adjacent orbits. Chaos can be identified simply by calculating the largest Lyapunov exponent, thereby determining whether nearby trajectories generally diverge $(\lambda>0)$ or converge $(\lambda<0)$. Any bounded motion in a system of at least one positive Lyapunov exponent is defined as chaotic, whereas nonpositive Lyapunov exponents indicate periodic motion. Numerous wellestablished algorithms are available for computing the Lyapunov spectrum of smooth dynamic systems [7-10]. However, these algorithms cannot be directly applied to 


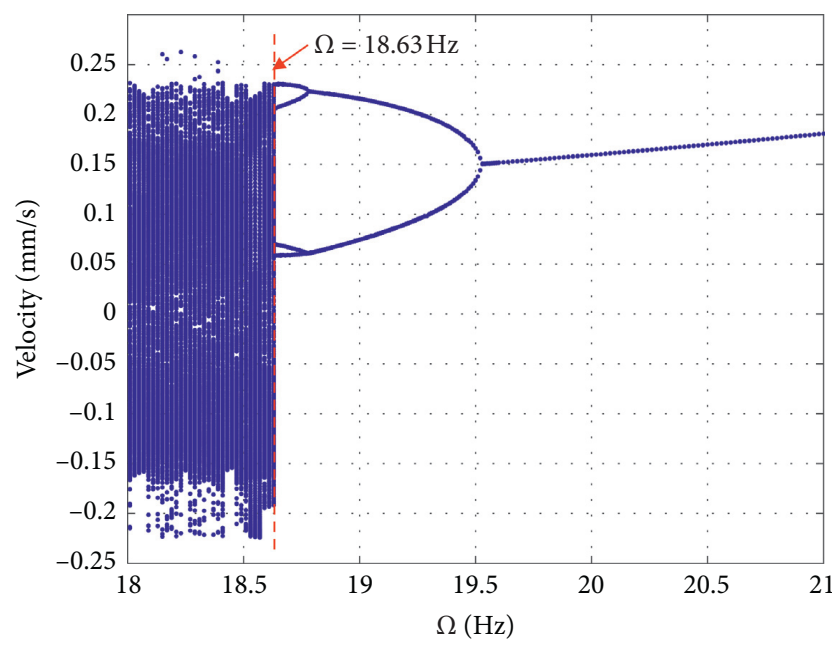

Figure 3: Bifurcation diagram of the system for $(A)_{0}=3.5 \mathrm{~V}$.

nonsmooth dynamic systems with discontinuities, such as dry friction, backlash, and saturation. In this study, we estimated the largest Lyapunov exponent in order to identify the onset of chaotic motion in AMB systems. Stefanski [13] proposed a simple method for estimating the largest Lyapunov exponent based on synchronization properties. Synchronization controls the response system by accessing the output of the drive system. Thus, the output of the response system asymptotically follows the output of the drive system. This method is described briefly in the following paragraph.

The dynamic system is decomposed into two subsystems, namely, a drive system

$$
\dot{u}=f(u)
$$

and a response system

$$
\dot{v}=f(v) \text {. }
$$

Consider a dynamic system that is composed of two identical $n$-dimensional subsystems; a coupling coefficient $d$ is applied only to the response system (5), while the drive system (4) remains unchanged. The first-order differential equations for such a system can be written as

$$
\begin{aligned}
\dot{u} & =f(u), \\
\dot{v} & =f(v)+d(u-v) .
\end{aligned}
$$

The condition of synchronization is given by the following inequality:

$$
d>\lambda_{\max }
$$

The smallest value of the coupling coefficient $d$ in the synchronization, $d_{s}$, is assumed to equal the largest Lyapunov exponent, as follows:

$$
d_{s}=\lambda_{\max }
$$

Equation (6) provides an augmented system, based on (1a)-(1d), as follows:

$$
\begin{aligned}
& \dot{u}_{1}=u_{2} \text {, } \\
& \dot{u}_{2}=-d_{1} u_{2}-d_{2} u_{1}+d_{3} u_{3}+d_{4} u_{4}+d_{5} u_{1}^{2}+d_{6} u_{1} u_{3} \\
& +d_{7} u_{1} u_{4}+d_{8} u_{3}^{2}+d_{9} u_{4}^{2}+d_{10} u_{1}^{3} \text {, } \\
& L \dot{u}_{3}+R u_{3}=K_{A}\left(e_{c 1 u}+A_{0} \sin \Omega t\right), \\
& L \dot{u}_{4}+R u_{4}=K_{A}\left(e_{c 2 u}+A_{0} \sin \Omega t\right), \\
& \dot{v}_{1}=v_{2}+d\left(u_{1}-v_{1}\right) \text {, } \\
& \dot{v}_{2}=-d_{1} v_{2}-d_{2} v_{1}+d_{3} v_{3}+d_{4} v_{4}+d_{5} v_{1}^{2}+d_{6} v_{1} v_{3} \\
& +d_{7} v_{1} v_{4}+d_{8} v_{3}^{2}+d_{9} v_{4}^{2}+d_{10} v_{1}^{3}+d\left(u_{2}-v_{2}\right) \text {, } \\
& L \dot{v}_{3}+R v_{3}=K_{A}\left(e_{c 1 v}+A_{0} \sin \Omega t\right)+d\left(u_{3}-v_{3}\right), \\
& L \dot{v}_{4}+R v_{4}=K_{A}\left(e_{c 2 v}+A_{0} \sin \Omega t\right)+d\left(u_{4}-v_{4}\right),
\end{aligned}
$$

where

$$
\begin{aligned}
& e_{c 1 u}=K_{H}\left(K_{P} u_{1}+K_{D} u_{2}+K_{I} \int u_{1} \mathrm{~d} t\right), \\
& e_{c 2 u}=K_{H}\left(-K_{P} u_{1}-K_{D} u_{2}-K_{I} \int u_{1} \mathrm{~d} t\right), \\
& e_{c 1 v}=K_{H}\left(K_{P} v_{1}+K_{D} v_{2}+K_{I} \int v_{1} \mathrm{~d} t\right), \\
& e_{c 2 v}=K_{H}\left(-K_{P} v_{1}-K_{D} v_{2}-K_{I} \int v_{1} \mathrm{~d} t\right),
\end{aligned}
$$

with the following limitations:

$$
\begin{aligned}
-3.5 \mathrm{~V} & <e_{c 1 u}<6.5 \mathrm{~V}, \\
-3.5 \mathrm{~V} & <e_{c 2 u}<6.5 \mathrm{~V} \\
0.0 \mathrm{~A} & <u_{3}<2.0 \mathrm{~A} \text { and } 0.0 \mathrm{~A}<u_{4}<2.0 \mathrm{~A}, \\
-3.5 \mathrm{~V} & <e_{c 1 v}<6.5 \mathrm{~V}, \\
-3.5 \mathrm{~V} & <e_{c 2 v}<6.5 \mathrm{~V}, \\
0.0 \mathrm{~A} & <v_{3}<2.0 \mathrm{~A} \text { and } 0.0 \mathrm{~A}<v_{4}<2.0 \mathrm{~A} .
\end{aligned}
$$

In the next step, the largest Lyapunov exponent of the system under consideration was determined for the chosen parametric values, in an aforementioned manner. Figure 8 is a plot of the results of numerical calculations and shows the estimated largest Lyapunov exponents obtained using the synchronization method. All of the largest Lyapunov exponents were positive with regard to the forcing frequency $(\Omega \leq 18.63 \mathrm{~Hz})$, indicating that the system exhibited chaotic motion. These results help enhance our understanding of the chaos phenomenon in AMB systems under rotation.

\section{Suppressing Chaos}

Learning to predict the behaviors of a chaotic system provides a number of benefits, with the ultimate objective being to assume control over the system. Improving the performance of a dynamic system necessitates chaotic motion to be 


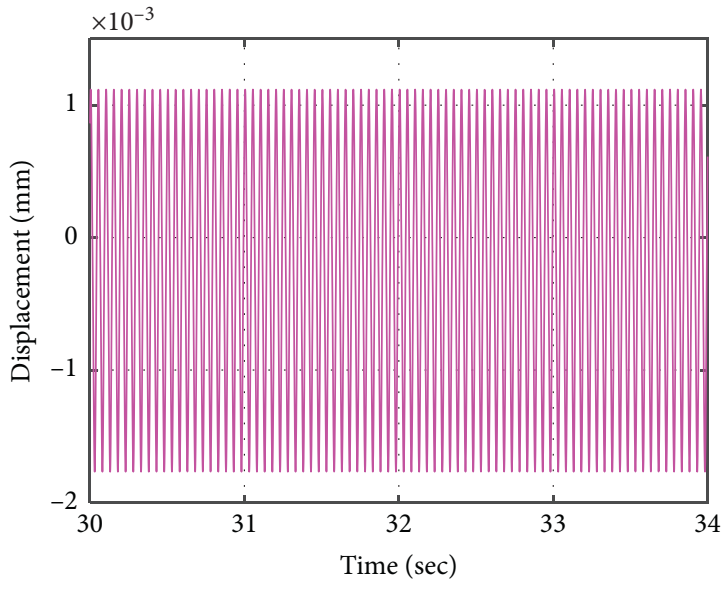

(a)

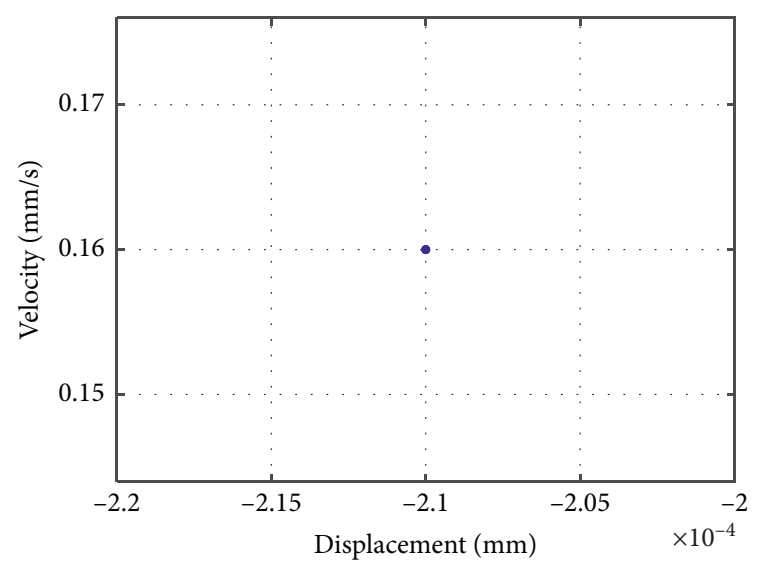

(c)

FIgure 4: Period-one orbit for $\Omega=20.0 \mathrm{~Hz}$ : (a) time response;

converted into a stable periodic orbit. Therefore, suitable control methods must be developed. Kapitaniak [18] and Pyragas [19] have proposed simple and effective timecontinuous control methods based on synchronization characteristics capable of converting chaotic motion into periodic motion. This method involves the construction of a feedback mechanism in conjunction with a specific timecontinuous perturbation. Figure 9 is a plot of the proposed feedback-controlled loop with an external periodic perturbation. The technique is explained briefly in the following paragraph.

Consider an $n$-dimensional dynamic system,

$$
\begin{aligned}
& \dot{p}=A(p), \\
& \dot{q}=B(q)+F(t),
\end{aligned}
$$

where $p(t), q(t) \in R^{n}$ denotes the state vector and $F(t)$ represents the input signal. System (14) without an input signal $(F(t)=0)$ is assumed to exhibit a strange attractor,

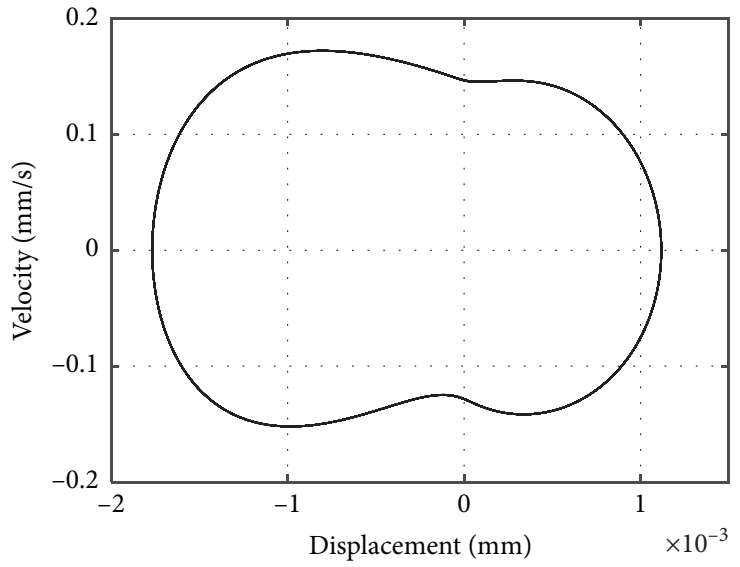

(b)

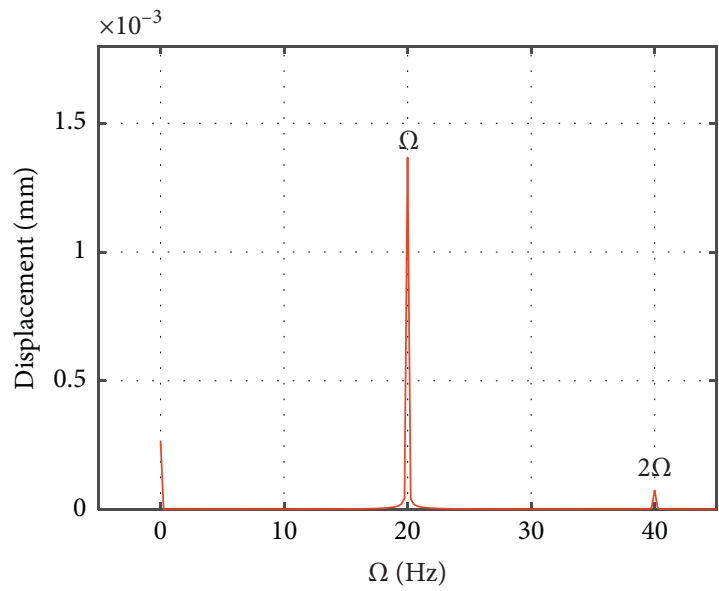

(d)

(b) phase portrait; (c) Poincaré map; (d) frequency spectrum.

whereas system (13) exhibits periodic motion. A periodic system is typically termed the drive system, whereas a chaotic system is called the response system. The strategy shown in Figure 9 is used to synchronize the two systems. The difference between signals $q(t)$ and $p(t)$ is utilized as a control signal:

$$
F(t)=K[q(t)-p(t)]
$$

where $K$ represents the feedback gain.

When $\Omega_{1}=20 \mathrm{~Hz}$ is selected in the drive system, the following equations reveal period-one motion.

$$
\begin{aligned}
\dot{p}_{1}= & p_{2}, \\
\dot{p}_{2}= & -d_{1} p_{2}-d_{2} p_{1}+d_{3} p_{3}+d_{4} p_{4}+d_{5} p_{1}^{2}+d_{6} p_{1} p_{3} \\
& +d_{7} p_{1} p_{4}+d_{8} p_{3}^{2}+d_{9} p_{4}^{2}+d_{10} p_{1}^{3}, \\
& L \dot{p}_{3}+R p_{3}=K_{A}\left(e_{c 1 p}+A_{0} \sin \Omega_{1} t\right),
\end{aligned}
$$




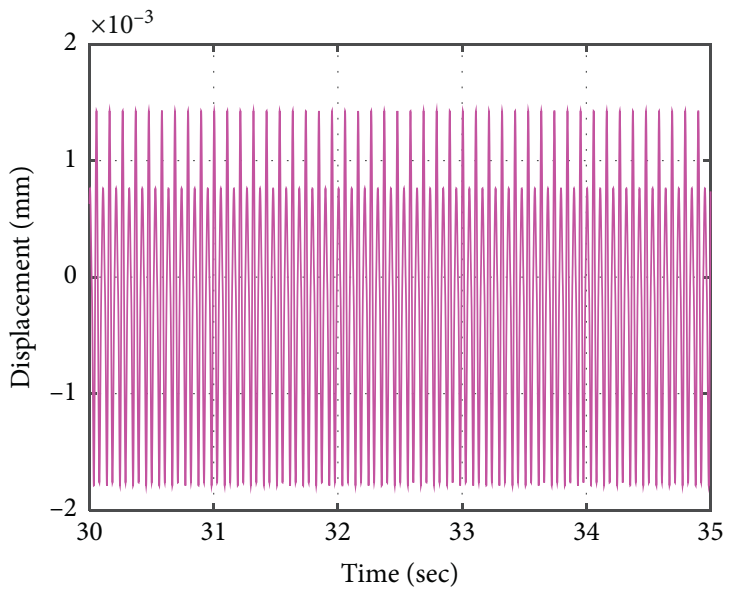

(a)

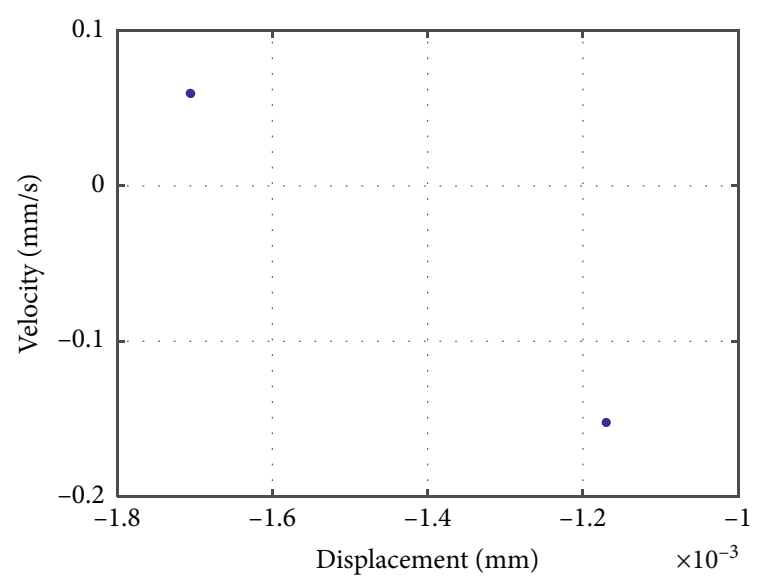

(c)

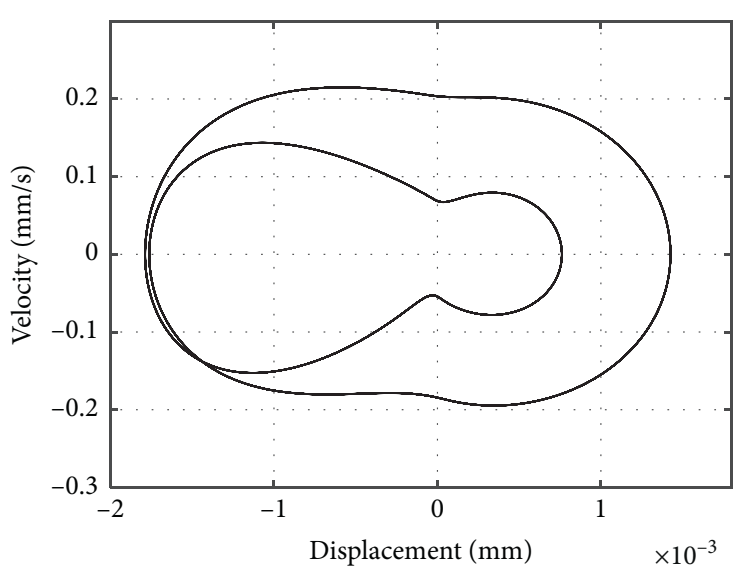

(b)

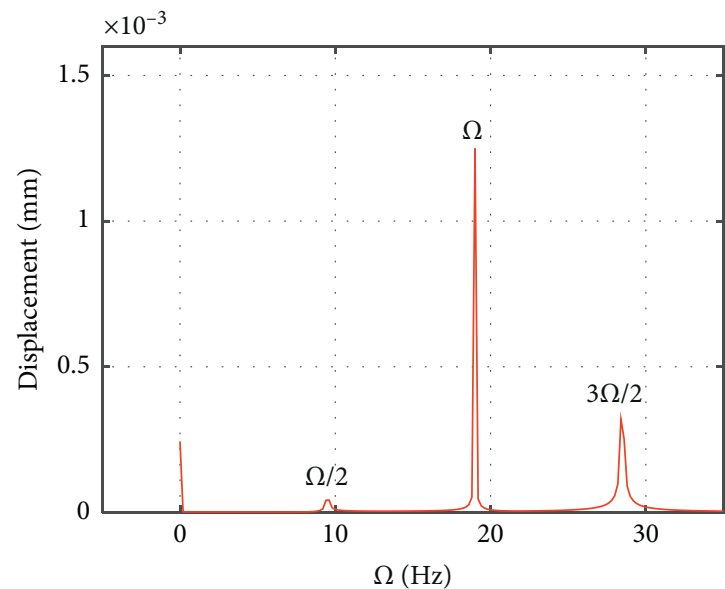

(d)

Figure 5: Period-two orbit for $\Omega=19.0 \mathrm{~Hz}$ : (a) time response; (b) phase portrait; (c) Poincaré map; (d) frequency spectrum.

$$
L \dot{p}_{4}+R p_{4}=K_{A}\left(e_{c 2 p}+A_{0} \sin \Omega_{1} t\right)
$$

where

$$
\begin{aligned}
& e_{c 1 p}=K_{H}\left(K_{P} p_{1}+K_{D} p_{2}+K_{I} \int p_{1} \mathrm{~d} t\right) \\
& e_{c 2 p}=K_{H}\left(-K_{P} p_{1}-K_{D} p_{2}-K_{I} \int p_{1} \mathrm{~d} t\right),
\end{aligned}
$$

with the following limitations:

$$
\begin{gathered}
-3.5 \mathrm{~V}<e_{c 1 p}<6.5 \mathrm{~V}, \\
-3.5 \mathrm{~V}<e_{c 2 p}<6.5 \mathrm{~V}, \\
0.0 \mathrm{~A}<p_{3}<2.0 \mathrm{~A}, \\
0.0 \mathrm{~A}<p_{4}<2.0 \mathrm{~A} .
\end{gathered}
$$

When $\Omega_{2}=18.2 \mathrm{~Hz}$ is selected in the response system, the following equations reveal chaotic motion:

$$
\dot{q}_{1}=q_{2}
$$

$$
\begin{gathered}
\dot{q}_{2}=-d_{1} q_{2}-d_{2} q_{1}+d_{3} q_{3}+d_{4} q_{4}+d_{5} q_{1}^{2}+d_{6} q_{1} q_{3} \\
+d_{7} q_{1} q_{4}+d_{8} q_{3}^{2}+d_{9} q_{4}^{2}+d_{10} q_{1}^{3}, \\
L \dot{q}_{3}+R q_{3}=K_{A}\left(e_{c 1 q}+A_{0} \sin \Omega_{2} t\right), \\
L \dot{q}_{4}+R q_{4}=K_{A}\left(e_{c 2 q}+A_{0} \sin \Omega_{2} t\right),
\end{gathered}
$$

where

$$
\begin{aligned}
& e_{c 1 q}=K_{H}\left(K_{P} q_{1}+K_{D} q_{2}+K_{I} \int q_{1} \mathrm{~d} t\right) \\
& e_{c 2 q}=K_{H}\left(-K_{P} q_{1}-K_{D} q_{2}-K_{I} \int q_{1} \mathrm{~d} t\right)
\end{aligned}
$$

with the following limitations: 


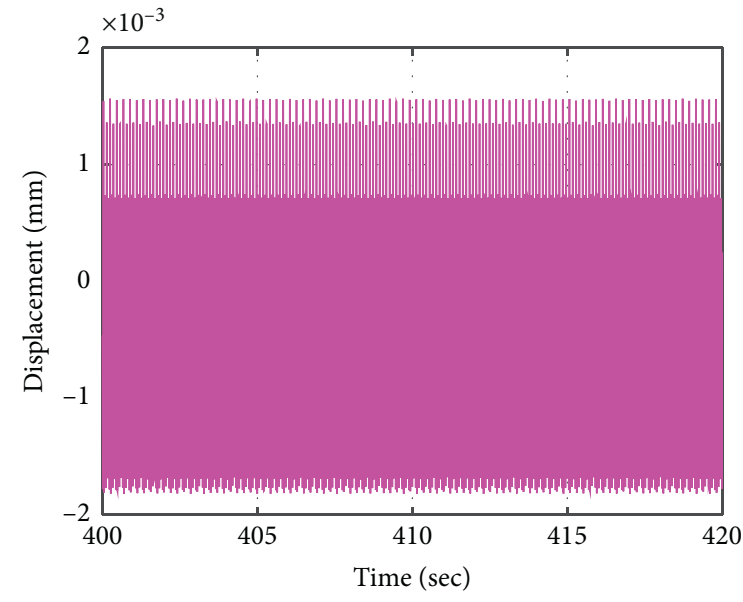

(a)

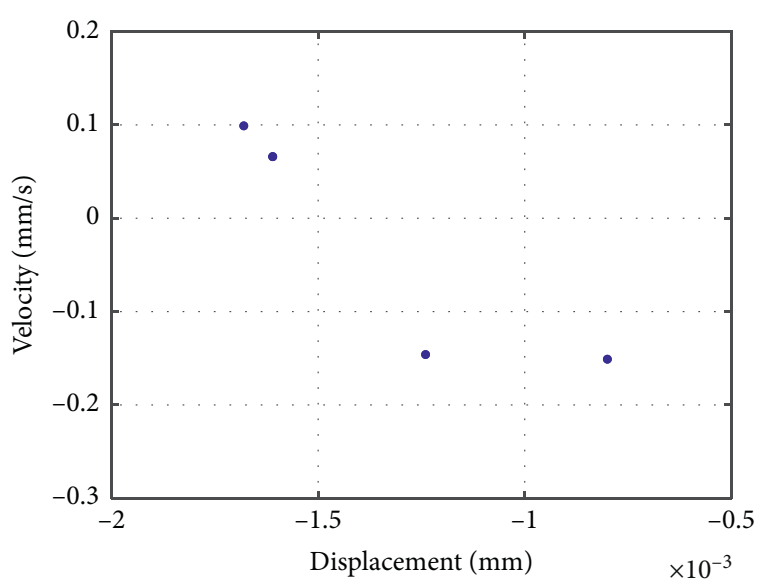

(c)

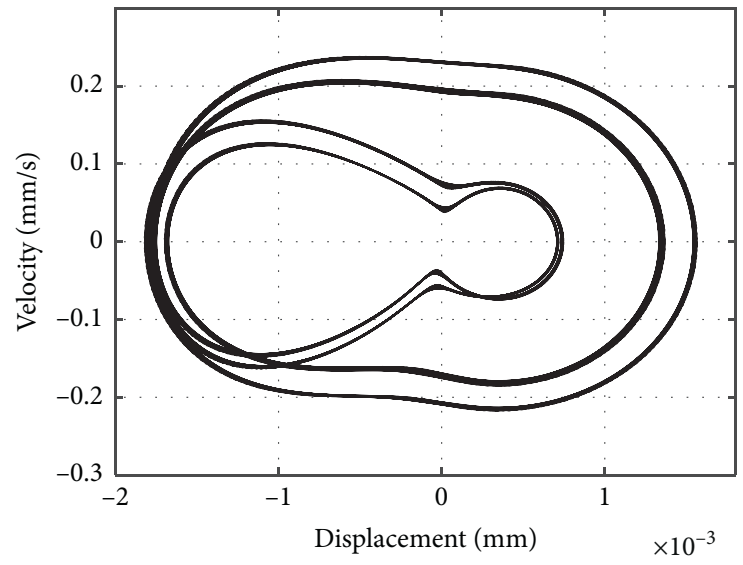

(b)

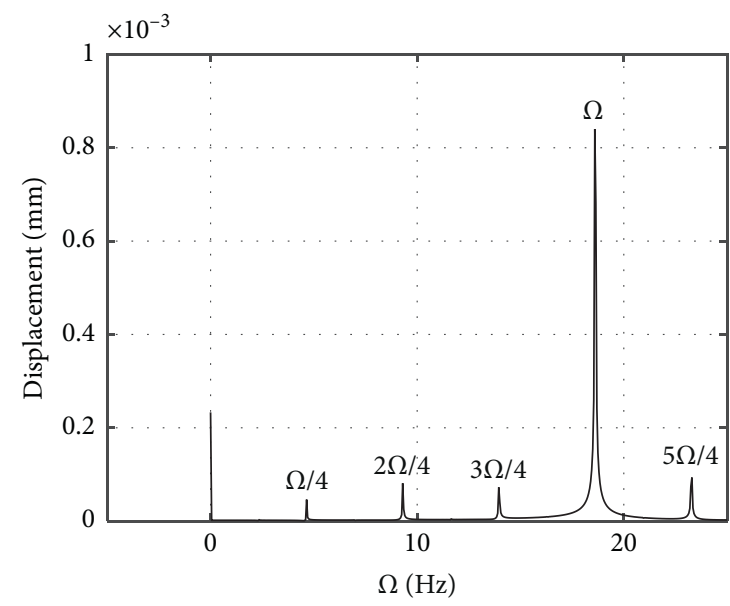

(d)

Figure 6: Period-four orbit for $\Omega=18.63 \mathrm{~Hz}$ : (a) time response; (b) phase portrait; (c) Poincaré map; (d) frequency spectrum.

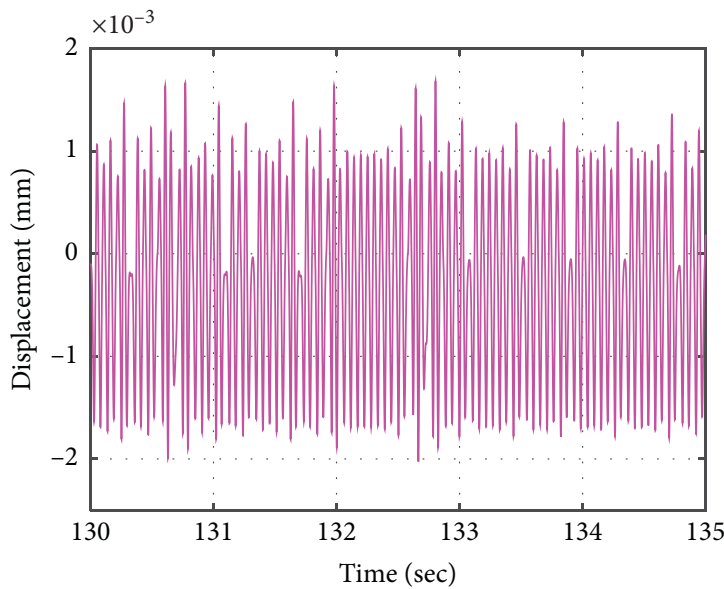

(a)

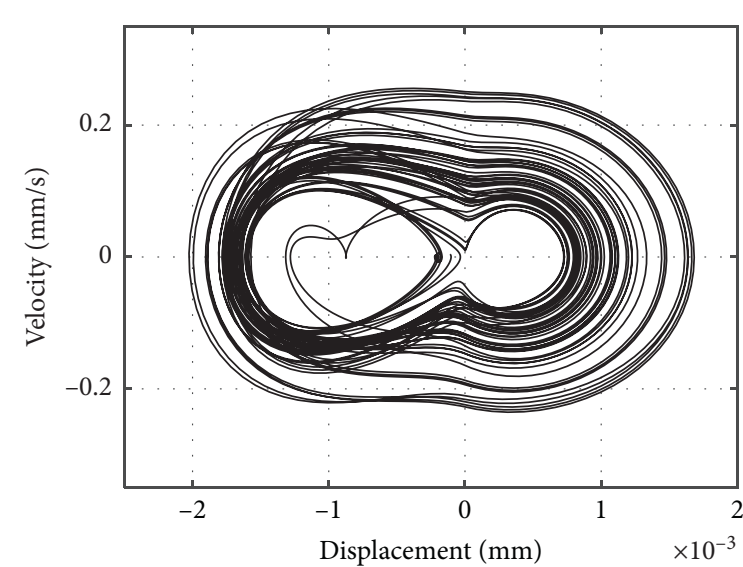

(b)

Figure 7: Continued. 


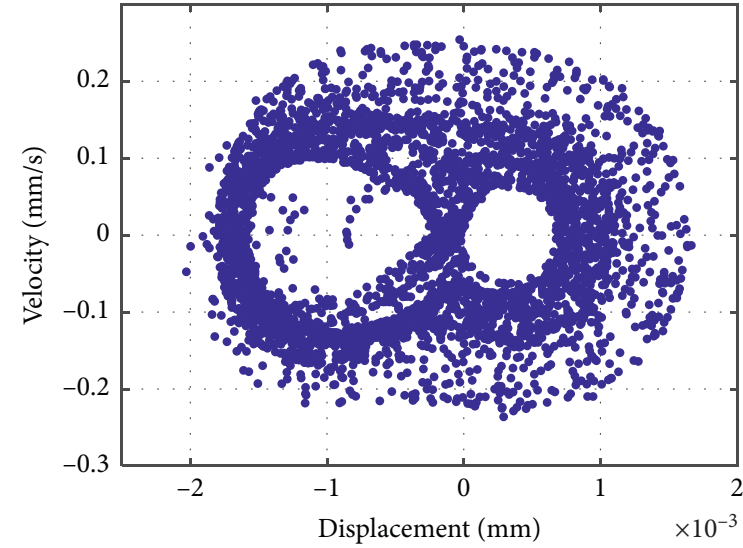

(c)

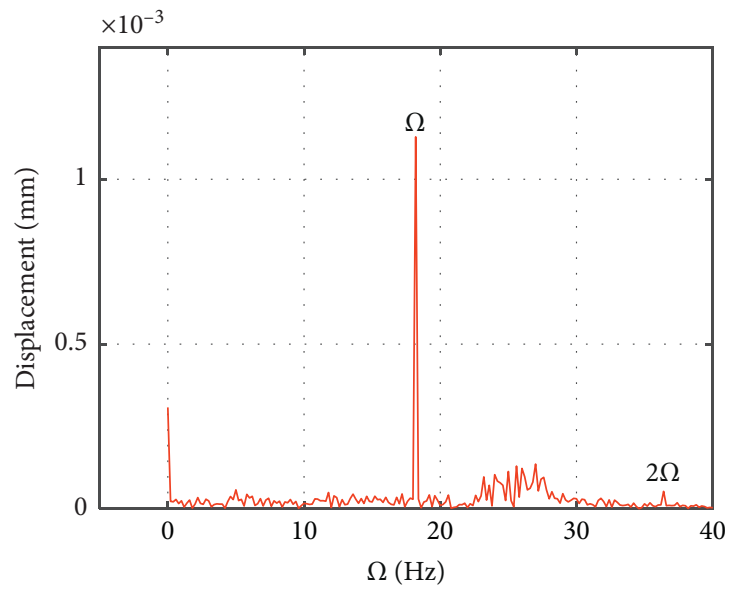

(d)

Figure 7: Chaotic motion for $\Omega=18.2 \mathrm{~Hz}$ : (a) time response; (b) phase portrait; (c) Poincaré map; (d) frequency spectrum.

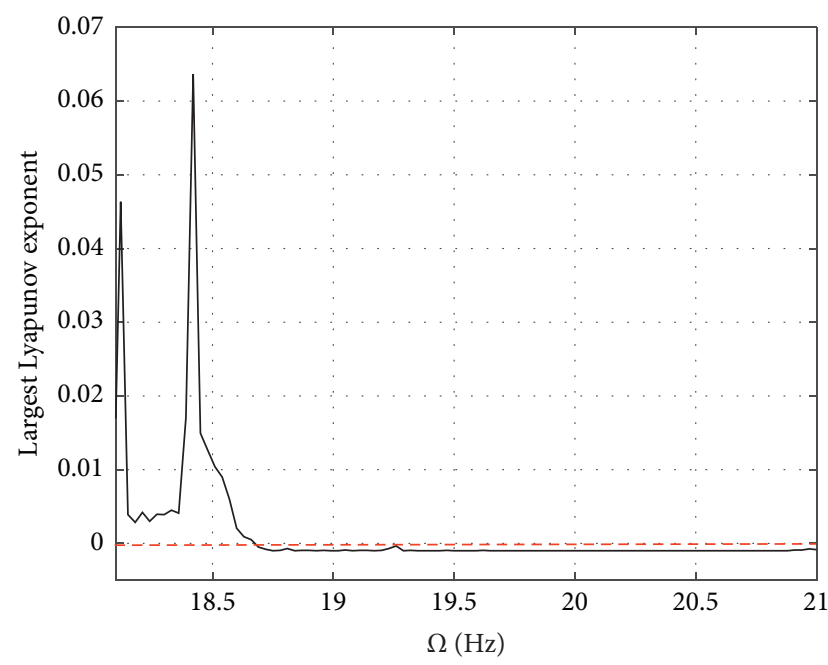

FIGURE 8: Evolutions of the largest Lyapunov exponent.

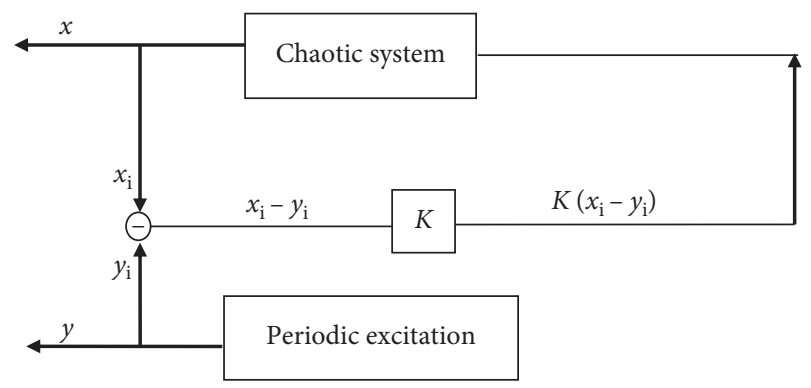

FIGURE 9: Block diagram of the continuous chaos control scheme.

$$
\begin{aligned}
-3.5 \mathrm{~V} & <e_{c 1 q}<6.5 \mathrm{~V}, \\
-3.5 \mathrm{~V} & <e_{c 2 q}<6.5 \mathrm{~V}, \\
0.0 \mathrm{~A} & <q_{3}<2.0 \mathrm{~A} \text { and } 0.0 \mathrm{~A}<q_{4}<2.0 \mathrm{~A} .
\end{aligned}
$$

Control signal (15) was introduced into (19a)-(19d) as a feedback control to synchronize (16a)-(16d) and (19a)-(19d). Control signal (15) was incorporated into (19a)-(19d), yielding the following coupled system, to achieve synchronization.

$$
\begin{aligned}
\dot{q}_{1}= & q_{2}+K\left(q_{1}-p_{1}\right), \\
\dot{q}_{2}= & -d_{1} q_{2}-d_{2} q_{1}+d_{3} q_{3}+d_{4} q_{4}+d_{5} q_{1}^{2}+d_{6} q_{1} q_{3} \\
& +d_{7} q_{1} q_{4}+d_{8} q_{3}^{2}+d_{9} q_{4}^{2}+d_{10} q_{1}^{3}+K\left(q_{2}-p_{2}\right), \\
L q_{3} & +R q_{3}=K_{A}\left(e_{c 1 q}+A_{0} \sin \Omega_{2} t\right)+K\left(q_{3}-p_{3}\right), \\
L \dot{q}_{4} & +R q_{4}=K_{A}\left(e_{c 2 q}+A_{0} \sin \Omega_{2} t\right)+K\left(q_{4}-p_{4}\right) .
\end{aligned}
$$

Equation (22a)-(22d) exhibited chaotic motion when $K=0$ and $\Omega_{2}=18.2 \mathrm{~Hz}$. The feedback gain $K$ was adjusted to be between 0.0 and -3000 to convert the dynamics of system (22a)-(22d) from chaotic to periodic motion. Figure 10 presents the resulting bifurcation diagram, which comprehensively explains the dynamic behavior of the system over a range of feedback gains. Figure 10 clarifies that chaotic motion occurred in the region between $K=0$ and $K=-120$. Stable periodic motion occurred when $K$ decreased to below -120 . When $K<-1000$, (22a)-(22d) display period-one motion. Accordingly, the synchronization was achieved in the AMB system by applying the control signal with $K<-1000$. When $t=10 \mathrm{~s}$, the chaotic AMB system (19a)-(19d) can be synchronized by applying the control signal with $K=-1300$, as shown in Figure 11. Thus, this chaotic system can be managed using the control signal $(K=-1300)$, such that chaotic motion is converted into period-one motion. Figure 11(a) presents the phase portrait of the controlled system, and Figure 11(b) plots the time response of displacement in which the synchronization control signal is injected after $10 \mathrm{~s}$. Figure 12 presents the associated synchronization errors, $e_{1}=q_{1}-p_{1}$ and $e_{2}=q_{2}-p_{2}$. When the synchronization errors equal zeros, 


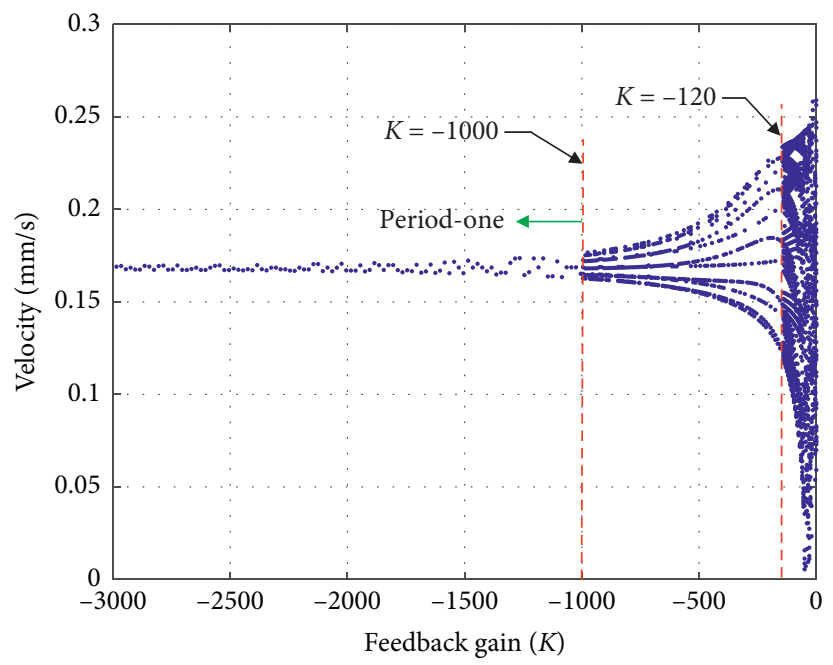

FIGURE 10: Bifurcation diagram of system with synchronization control, where $K$ denotes feedback gain.

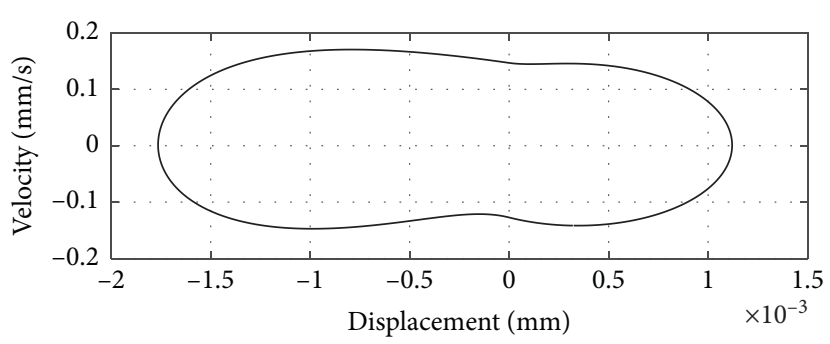

(a)

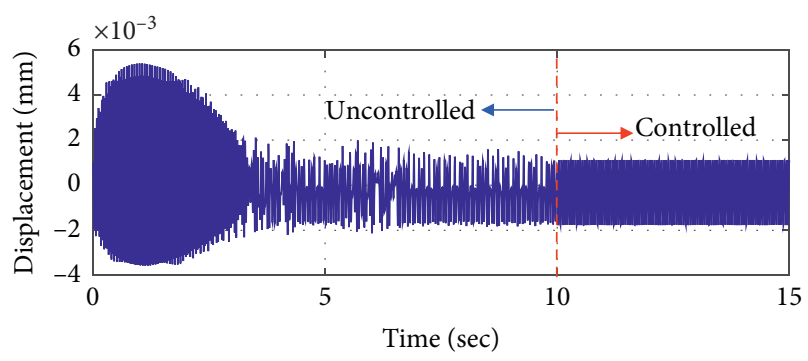

(b)

FIGURE 11: Transforming chaotic motion into period-one orbit at $K=-1300$ and $\Omega=18.2 \mathrm{~Hz}$ : (a) phase portrait of controlled system; (b) time responses of displacement. Synchronization control signal is introduced after $10 \mathrm{~s}$.

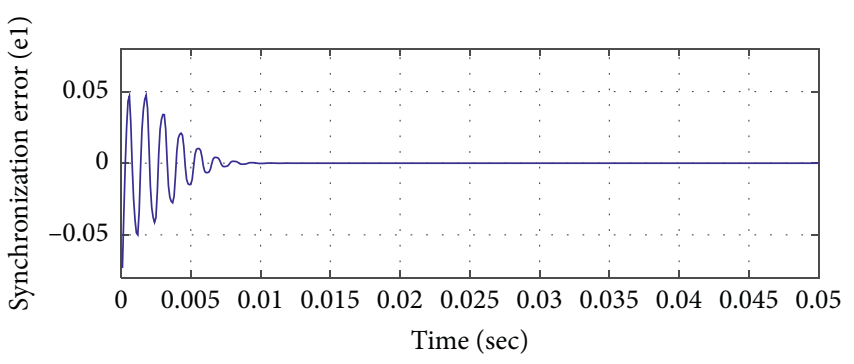

(a)

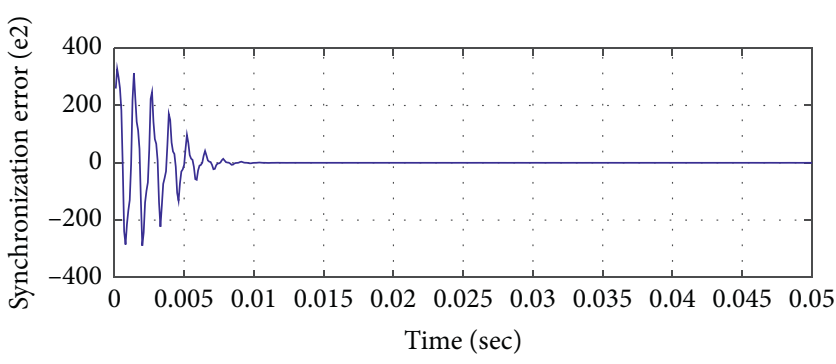

(b)

FIgURE 12: Synchronization errors associated with controlled system at $K=-1300$.

the synchronization is achieved. The control signal controlled the chaotic system, which converged to a stable equilibrium point. Therefore, the synchronization control method successfully suppressed chaotic motion and improved the chatter behavior of the AMB system. Thus, the performance of the AMB system could be improved considerably at high-speed rotations.

\section{Conclusions}

This work used an identified nonlinear model to explore global bifurcations and chaos control problem in AMB systems. Dynamic behaviors over the entire range of parameter values could be observed in a bifurcation diagram, which revealed that AMB systems exhibit period-doubling bifurcations and chaotic motions. The largest Lyapunov exponent, derived using the properties of synchronization, provided the most powerful approach for analyzing chaotic motion in a system. Controlling chaotic motion is an effective method to prevent chatter vibration in AMB systems. We propose a continuous feedback control method based on synchronization properties to suppress chaotic motion in AMB systems. Our analysis revealed that the proposed nonlinear model could predict the occurrence of global 
bifurcations and chaos control in AMB systems. These results indicate that the proposed system has potential for application across a wide range of functions in the design of magnetic levitation systems. Furthering the development of magnetically levitated vehicles requires an understanding of their dynamic characteristics from the viewpoint of stability, safety, and ride quality at high speeds. We believe that studying the dynamics and controlling chaotic vibrations in the magnetically levitated systems will help to advance the development of magnetic transportation systems.

\section{Data Availability}

All data generated or analyzed during this study are included within this article.

\section{Conflicts of Interest}

The author declares that there are no conflicts of interest regarding the publication of this paper.

\section{Acknowledgments}

This research was supported by the Ministry of Science and Technology in Taiwan, Republic of China, under project number MOST 108-2221-E-212-010-MY3.

\section{References}

[1] S. C. Chang and P. C. Tung, "Nonlinear identification of a magnetic bearing system with closed loop control," JSME International Journal Series C, vol. 42, no. 4, pp. 982-990, 1999.

[2] W. Zhang and X. P. Zhan, "Periodic and chaotic motions of a rotor-active magnetic bearing with quadratic and cubic terms and time-varying stiffness," Nonlinear Dynamics, vol. 41, no. 4, pp. 331-359, 2005.

[3] W. Zhang, J. W. Zu, and F. X. Wang, "Global bifurcations and chaos for a rotor-active magnetic bearing system with timevarying stiffness," Chaos, Solitons \& Fractals, vol. 35, no. 3, pp. 586-608, 2008.

[4] A. K. Mando, D. Yemélé, W. T. Sokamte, and A. Fomethe, "Structural static stability and dynamic chaos of active electromagnetic bearing systems: analytical investigations and numerical simulations," Journal of Vibration and Control, vol. 24, no. 24, pp. 5774-5793, 2018.

[5] W. S. Ma, W. Zhang, and Y. F. Zhang, "Stability and multipulse jumping chaotic vibrations of a rotor-active magnetic bearing system with 16-pole legs under mechanical-electricelectromagnetic excitations," European Journal of MechanicsA/Solids, vol. 85, Article ID 104120, 2021.

[6] A. Kandil, M. Sayed, and N. A. Saeed, "On the nonlinear dynamics of constant stiffness coefficients 16-pole rotor active magnetic bearings system," European Journal of Mechanics-A/ Solids, vol. 84, Article ID 104051, 2020.

[7] I. Shimada and T. Nagashima, "A numerical approach to ergodic problem of dissipative dynamical systems," Progress of Theoretical Physics, vol. 61, no. 6, pp. 1605-1616, 1979.

[8] A. Wolf, J. B. Swift, H. L. Swinney, and J. A. Vastano, "Determining lyapunov exponents from a time series," Physica D: Nonlinear Phenomena, vol. 16, no. 3, pp. 285-317, 1985.

[9] G. Benettin, L. Galgani, A. Giorgilli, and J.-M. Strelcyn, "Lyapunov Characteristic Exponents for smooth dynamical systems and for Hamiltonian systems; a method for computing all of them. part 1: Theory," Meccanica, vol. 15, no. 1, pp. 9-20, 1980.

[10] G. Benettin, L. Galgani, A. Giorgilli, and J.-M. Strelcyn, "Lyapunov Characteristic Exponents for smooth dynamical systems and for Hamiltonian systems; A method for computing all of them. part 2: numerical application," Meccanica, vol. 15 , no. 1 , pp. 21-30, 1980.

[11] P. C. Müller, "Calculation of lyapunov exponents for dynamic systems with discontinuities," Chaos, Solitons \& Fractals, vol. 5, no. 9, pp. 1671-1681, 1995.

[12] N. Hinrichs, M. Oestreich, and K. Popp, "Dynamics of oscillators with impact and friction," Chaos, Solitons \& Fractals, vol. 8, no. 4, pp. 535-558, 1997.

[13] A. Stefanski, "Estimation of the largest Lyapunov exponent in systems with impacts," Chaos, Solitons \& Fractals, vol. 11, no. 15 , pp. 2443-2451, 2000.

[14] E. Ott, C. Grebogi, and J. A. Yorke, "Controlling chaos," Physical Review Letters, vol. 64, no. 11, pp. 1196-1199, 1990.

[15] I. M. Ginarsa, A. Soeprijanto, and M. H. Purnomo, "Controlling chaos and voltage collapse using an ANFIS-based composite controller-static var compensator in power systems," International Journal of Electrical Power \& Energy Systems, vol. 46, pp. 79-88, 2013.

[16] Z. Shen and J. Li, "Chaos control for a unified chaotic system using output feedback controllers," Mathematics and Computers in Simulation, vol. 132, pp. 208-219, 2017.

[17] S. M. A. Pahnehkolaei, A. Alfi, and J. A. Tenreiro Machado, "Chaos suppression in fractional systems using adaptive fractional state feedback control," Chaos, Solitons \& Fractals, vol. 103, pp. 488-503, 2017.

[18] T. Kapitaniak, "Continuous control and synchronization in chaotic systems," Chaos, Solitons \& Fractals, vol. 6, pp. 237244, 1995.

[19] K. Pyragas, "Continuous control of chaos by self-controlling feedback,” Physics Letters A, vol. 170, no. 6, pp. 421-428, 1992.

[20] X. Yi, R. Guo, and Y. Qi, "Stabilization of chaotic systems with both uncertainty and disturbance by the UDE-based control method," IEEE Access, vol. 8, pp. 62471-62477, 2020.

[21] N. A. Saeed and A. Kandil, "Two different control strategies for 16-pole rotor active magnetic bearings system with constant stiffness coefficients," Applied Mathematical Modelling, vol. 92, pp. 1-22, 2021.

[22] S. C. Chang, "Nonlinear dynamics and suppressing chaos in magnetic bearing system," Mathematical Problems in Engineering, vol. 2020, Article ID 6669941, 10 pages, 2020.

[23] IMSL, Inc., User's Manual-IMSL Math/Library, Valby, Denmark, 1989. 